\title{
THE FLUX OF HARD RADIATION FROM M87
}

\author{
G. J. FISHMAN, F. R. HAR NDEN, Jr., and R. C. HAYMES \\ Dept. of Space Science, William Marsh Rice University, Houston, Texas, U.S.A.
}

This paper reports upon an observation of hard X-radiation from the direction of M87. Earlier (Haymes et al., 1968a), we reported the apparent detection of hard radiation from the general direction of Virgo; the experiment discussed here, conducted 16 months later, recorded approximately the same flux from a smaller region of sky that tends to isolate M87 as the most likely candidate for the source.

Other groups (Adams et al., 1969; Bradt et al., 1967; Friedman and Byram, 1967) have detected soft X-radiation (photon energies between $\sim 1$ and $\sim 10 \mathrm{keV}$ ) from M87. The present observation, conducted at energies greater than $30 \mathrm{keV}$, recorded additional evidence for a flux in agreement with an extrapolation of the power law that fits both the core radio spectrum and the soft X-ray flux from the radiogalaxy.

Most of the balloon-borne instrumentation used in the present work was the same as previously described (Haymes et al., 1968b), with the exception of the detector itself. An additional active collimator was inserted in the beam in front of the actively collimated detector previously used and the assembly was flown as a narrow-angle device. The thickness of the additional collimator was nine inches of plastic scintillator (Nuclear Enterprises Corporation Type NE-102). It was viewed by four additional photomultipliers connected in anti-coincidence with the photomultiplier viewing the central NaI(T1) crystal.

This thickness of plastic corresponds to at least 3.5 mean free paths of absorber at photon energies up to $100 \mathrm{keV}$. The additional collimator resulted in a half-flux angle that was $3^{\circ}$ from the axis, at energies less than $100 \mathrm{keV}$. The purpose of this improvement in the angular resposne was to enable M87 to be isolated from $3 \mathrm{C} 273$, one of the other candidates for the source of hard radiation previously found from Virgo.

The balloon was launched from Palestine, Texas, at 0305 CST on December 11, 1968. It floated at a pressure altitude that averaged $3.2 \mathrm{mb}$ for 5.2 hours, before termination occurred at 1110 CST. The pressures were measured with a Metrophysics Corporation transducer that senses the thermal conductivity of the atmosphere, as well as by aneroid devices.

Good telemetered data were received throughout the flight; none had to be discarded because of noise. We have rejected in the analysis the three slightly discordant background segments that were widely separated in time, along with their associated M87 segments. Hence the analysis is based on $133 \mathrm{~min}$ of Virgo data and approximately 118 min of background measurements; no known discrete sources of $\mathrm{X}$ radiation were contained in the background data used in the analysis. It should be noted that the conclusions reached herein are essentially unaffected if all of the segments rejected are instead included in the analysis.

As in the previous experiment, the detector was continuously rotated about the 
polar axis at the sidereal rate, so that it would track M87 in hour angle. The azimuth of the polar shaft was automatically changed by $180^{\circ}$ every $10.5 \mathrm{~min}$ after the azimuth servo system was turned on at 0440 CST. Background measurements were taken during each of the alternate segments when the detector was turned away from Virgo.

While the azimuth of the polar axis returns to the same angle (i.e., South) during each background segment, the detector azimuth does not do so, because of the continuous rotation. Hence the background was measured for a range of azimuths. The region of sky scanned in the background portions of the flight discussed here consisted of an arc of disconnected segments, each 10.5 min long and $6^{\circ}$ wide (with equal exposures to the East and to the West because of the 0730 transit time of M87). The angular separation of the segments from M87 is twice the zenith angle of M87; the separation varied during the flight from $40^{\circ}$ at meridian transit to approximately $90^{\circ}$ near the beginning and end of the observing period.

As before, the average of the two background segments that were time-contiguous with an M87 segment was compared with the flux measured during that M87 segment. The results discussed below are inconsistent with a significant azimuthal variation of the photon flux. In addition, this technique permits corrections to be made for other background changes that may occur, such as those caused by changes in the zenith angle, altitude or latitude. The sensitivity of the method is shown by the upper limits found for Centaurus A (Haymes et al., 1969). The upper limit set in that experiment at the $95 \%$ confidence level amounts to $0.7 \%$ of the background in the $40-100 \mathrm{keV}$ interval; any background asymmetry is smaller than that.

The radiation from Virgo was found by averaging the background segments adjacent in time to a Virgo observation period and then subtracting this average from the data obtained while M87 was in the field of view. A residual was thus formed for each Virgo period.

Each residual was corrected for atmospheric absorption caused by the air along the line of sight between M87 and the detector. It was also corrected for pointing errors; the narrower beam-width employed in the present experiment increased the importance of the pointing error, which was mainly due to the variation in geomagnetic declination over the balloon's trajectory. Corrections for absolute efficiency and the escape of $\mathrm{K}$ X-radiation were applied as were the corrections for the absorption caused by the central photomultiplier, etc. (Haymes et al., 1968b). These latter correction-factors were experimentally determined in our laboratory prior to the experiment. No gain change as great as \pm 1 channel was detected in the 128 channel pulse-height analysis conducted during the flight.

Figure 1 shows the results of the flight, corrected as outlined above. All pulse height channels are displayed, as well as the 13-channel sums that were formed in order to improve the statistics. No flux is observed above $100 \mathrm{keV}$; upper limits may be assigned to the higher energy radiation from M87. A small flux is seen in the energy interval $40-95 \mathrm{keV}$; it is 2.8 standard deviations $(2.8 \sigma)$ above the background at those energies.

The excess counting rate from M87 is constant to within statistics throughout the 
flight. This constancy, observed over a range of East-West and North-South azimuths, indicates that the observed flux is not due to an azimuthal asymmetry. Also, the increase is $\sim 4$ times any background asymmetry, as observed in the Centaurus A experiment, which makes it seem likely that the $40-100 \mathrm{keV}$ enhanced flux is real and extraterrestrial.

This flux at the top of the earth's atmosphere from the direction of M87 is
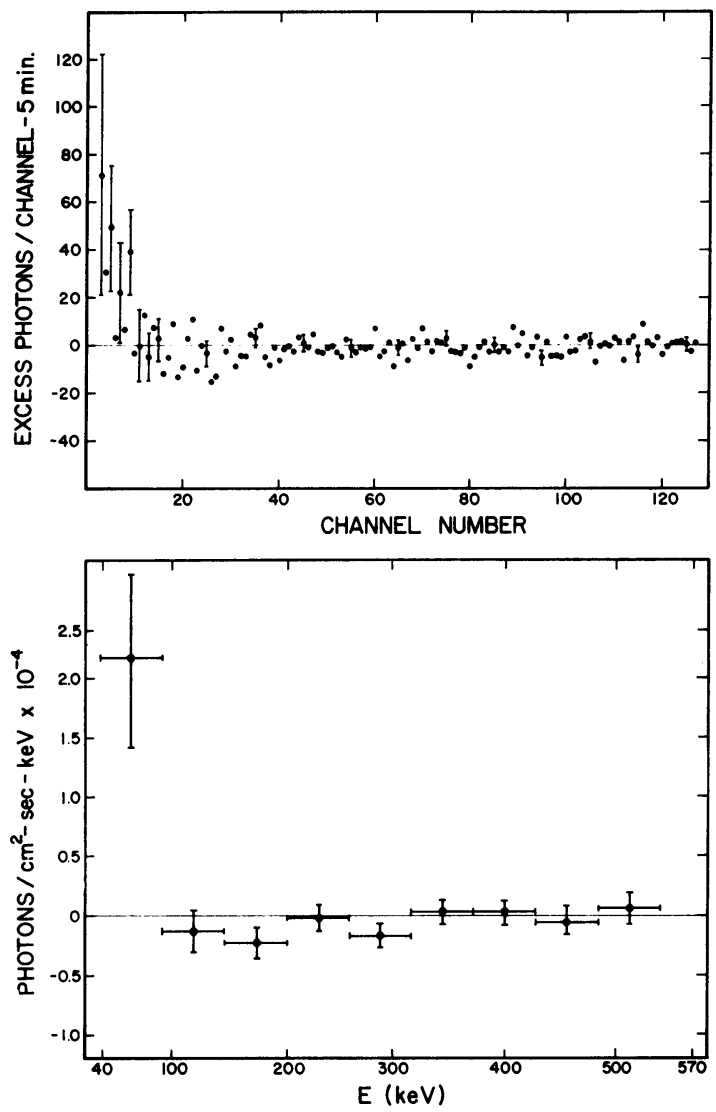

Fig. 1. Upper plot is the observed excess from the M87 region for each pulse height channel over the entire flight. The method of background subtraction is described in the text. Lower plot is the derived flux for 13-channel sums at the top of the earth's atmosphere. Error bars shown are \pm one standard deviation for both plots.

$2.13 \pm 0.75 \times 10^{-4}$ photons $/ \mathrm{cm}^{2}$-sec-keV. It is to be compared with the value of $1.22 \pm 0.52 \times 10^{-4}$ photons $/ \mathrm{cm}^{2}-\mathrm{sec}-\mathrm{keV}$ we previously reported for Virgo. The larger error in the present experiment is caused by the importance of the pointing error when using a narrow-beam device.

To within statistics, the flux agrees with that observed on August 10, 1967. If we assume M87 to be the source of hard radiation observed on both occasions and further assume that M87 has not varied its emission at these wavelengths during this period, 
we may combine the results of the two measurements. The weighted average of the two is $3.5 \sigma$ above background; it is $1.51 \pm 0.43 \times 10^{-4}$ photons $/ \mathrm{cm}^{2}-\mathrm{sec}-\mathrm{keV}$, in the energy interval $40-100 \mathrm{keV}$, at the top of the earth's atmosphere. It would appear from the agreement of the two measurements, that 3C273 contributed little if any radiation to the flux found earlier.

These results on M87 do not agree with those reported by McClintock et al. (1969). Those investigators failed to detect radiation at energies up to $100 \mathrm{keV}$ from M87 with a proportional-counter balloon-borne experiment conducted on October 25 , 1968; they reported upper limits for the flux and concluded that a break exists in the M87 spectrum at energies between those of soft X-radiation and their threshhold at $\sim 20 \mathrm{keV}$. While it is possible that M87 is a variable source, the difference most likely arises in the manner by which the background is determined. Specifically, we suggest that perhaps the constant-azimuth method ( $36^{\circ}$ East and West from M87) and/or the

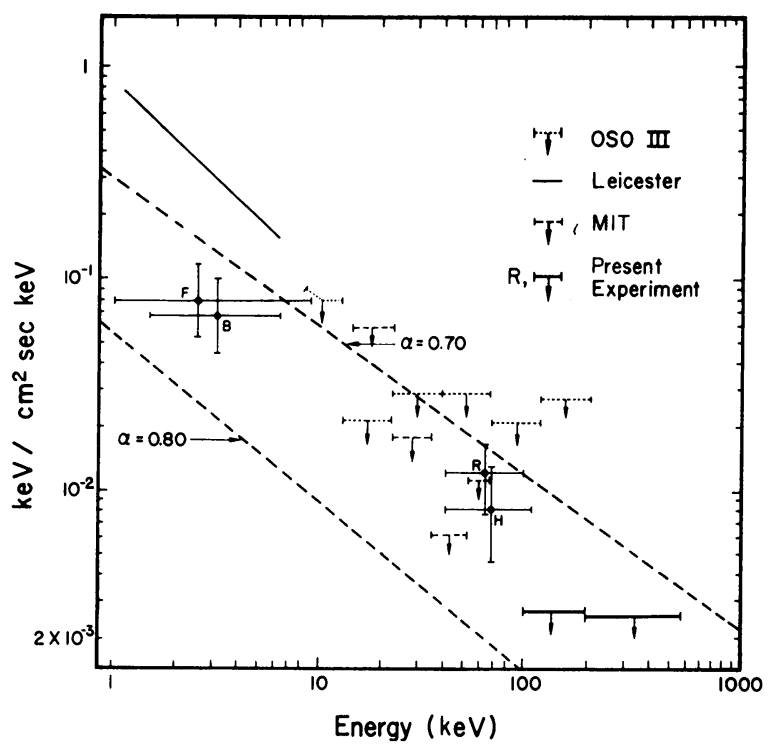

Fig. 2. Measurements of M87 in the X-ray region. Data plotted are from the following references: 'F', Friedman and Byram (1967); 'B', Bradt et al. (1967); 'Leicester', Adams et al. (1969); 'MIT', McClintock et al. (1969); 'OSO III', Hudson et al. (1968), and 'H', Haymes et al. (1968). Also shown are two possible extrapolations of the radio core spectrum, indicated by values of their spectral index, $\alpha$.

Upper limits indicated are at the $2 \sigma$ level; error bars associated with positive fluxes are $\pm 1 \sigma$.

pulse-shape discrimination (PSD) technique used by McClintock et al. (1969) may be responsible for the difference. The sun, observed to exhibit two Importance One flares during their flight (ESSA, 1968), was only $34^{\circ}$ from M87 and was closer than that to the field of view of their detector during their west background observations. Solar flares are known to have hard solar radiation associated with them; hard Xradiation was in fact detected from the sun during both of the flares in question by the Explorer 37 satellite (ESSA, 1968). Also, if the PSD technique is not $100 \%$ 
effic ient in rejecting charged particles, any slight East-West asymmetry in the chargedparticle spectrum could give rise to the null result reported by those investigators. The experiment reported here was conducted when the sun was about $75^{\circ}$ away from M87 and during a time of relative solar calm (ESSA, 1969).

Figure 2 shows, however, that there is no great inconsistency between the two measurements at the $2 \sigma$ level. We also show in Figure 2 the $2 \sigma$ upper limits we have deduced from our data at higher energies. At $506 \mathrm{keV}$, the upper limit on the redshifted positron annihilation flux is $1 \times 10^{-3}$ photons $\mathrm{cm}^{-2} \mathrm{sec}^{-1}$, and the $2 \sigma$ upper limit on the 'overflow counting rate' (i.e., those pulses due to photons with energies greater than $570 \mathrm{keV}$ ) is $6 \times 10^{-4}$ counts $/ \mathrm{cm}^{2}$-sec.

Also shown in Figure 2 are two extrapolations into the X-ray region of the core radio power law spectrum. The upper limits established on the flux by the present experiment at high energies are consistent with a spectral steepening above $100 \mathrm{keV}$. However, a single power law, presumably synchrotron in origin, may be fit to the data on the radiation in the radio and soft X-ray regions of the spectrum, as well as to the 40-100 keV flux; such a curve is also consistent with the high-energy upper limits found in the present work. The several optical determinations that have been made are in disagreement.

Felten et al. (1969) have found that the spectral index of the best-fit power law is 0.75 . Since the spectrum extends to at least $40 \mathrm{keV}$, it would appear that M87 is most luminous in the hard X-ray region of the spectrum.

\section{Acknowledgments}

We wish to express our thanks to Mr. A. C. Heath, who constructed much of the apparatus and helped during the flight. The research was supported in part by Project Themis under Contract N00014-68-A-0503 and in part by the Air Force Office of Scientific Research, United States Air Force, under Contract F44620-69-C-0083.

\section{References}

Adams, D. J., Cooke, B. A., Evans, K., and Pounds, K. A.: 1969, Nature 222, 759.

Bradt, H., Mayer, W., Naranan, S., Rappaport, S., and Spads, G.: 1967, Astrophys. J. (Letters) 150 , L199.

ESSA, Solar-Geophysical Data, Reports No. IER-FB-291, 292: 1968, Boulder, Colo., Environmental Science Service Administration.

ESSA, Solar-Geophysical Data, Report No. IER-FB-293: 1969, Boulder, Colo., Environmental Science Service Administration.

Felten, J. E., Arp, H. C., and Lynds, C. R. : 1969, Contributions from the Kitt Peak National Observatory, No. 390.

Friedman, H. and Byram, E.: 1967, Science 158, 257.

Haymes, R. C., Ellis, D. V., Fishman, G. J., Kurfess, J. D., and Tucker, W. H.: 1968a, Astrophys. J. (Letters) 151, L9.

Haymes, R. C., Ellis, D. V., Fishman, G. J., Glenn, S. W., and Kurfess, J. D.: 1968b, Astrophys. J. (Letters) 151, L131.

Hudson, H. S., Peterson, L. E., and Schwartz, D. A.: 1969, Solar Phys. 6, 205.

McClintock, J. E., Lewin, W. H. G., Sullivan, R. J., and Clark, G. W.: 1969, Massachusetts Institute of Technology Preprint Number CSR-P-69-13. Submitted to Nature. 